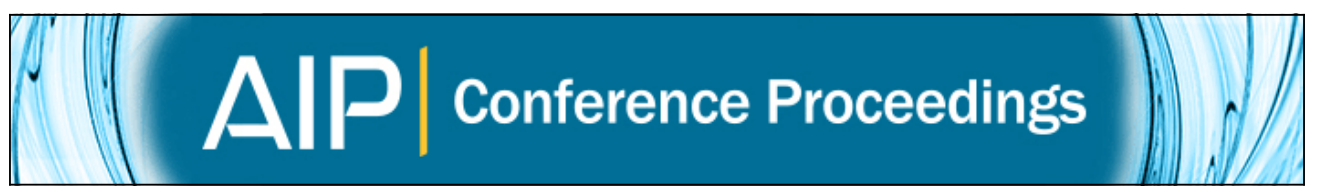

\title{
Nuclear Physics and the New Standard Model
}

Michael J. Ramsey-Musolf

Citation: AIP Conference Proceedings 1265, 285 (2010); doi: 10.1063/1.3480187

View online: http://dx.doi.org/10.1063/1.3480187

View Table of Contents:

http://scitation.aip.org/content/aip/proceeding/aipcp/1265?ver=pdfcov

Published by the AIP Publishing

\section{Articles you may be interested in}

Recent results in particle and nuclear physics from lattice QCD

AIP Conf. Proc. 1441, 49 (2012); 10.1063/1.3700480

Forward Physics at the LHC: within and beyond the Standard Model

AIP Conf. Proc. 1038, 95 (2008); 10.1063/1.2987191

Probing beyond the Standard Model with Muons

AIP Conf. Proc. 981, 97 (2008); 10.1063/1.2899012

Cosmology and Physics beyond the Standard Model

AIP Conf. Proc. 910, 3 (2007); 10.1063/1.2752478

Standard-Model Tests with Superallowed $\beta$ Decay: Nuclear Data Applied to Fundamental Physics

AIP Conf. Proc. 769, 678 (2005); 10.1063/1.1945100 


\title{
Nuclear Physics and the New Standard Model
}

\author{
Michael J. Ramsey-Musolf \\ Department of Physics, University of Wisconsin-Madison, Madison, WI 53706 USA \\ and \\ Kellogg Radiation Laboratory, California Institute of Technology, Pasadena, CA 91125 USA
}

\begin{abstract}
Nuclear physics studies of fundamental symmetries and neutrino properties have played a vital role in the development and confirmation of the Standard Model of fundamental interactions. With the advent of the CERN Large Hadron Collider, experiments at the high energy frontier promise exciting discoveries about the larger framework in which the Standard Model lies. In this talk, I discuss the complementary opportunities for probing the "new Standard Model" with nuclear physics experiments at the low-energy high precision frontier.
\end{abstract}

Keywords: Symmetries, Physics Beyond the Standard Model

PACS: $43.35 . \mathrm{Ei}, 78.60 . \mathrm{Mq}$

\section{INTRODUCTION}

The Standard Model (SM) of fundamental interactions is one of the triumphs of 20th century physics. It utilizes a simple and elegant symmetry principle to explain both a wide variety of laboratory observations as well as astrophysical phenomena. Much of the discussion at this Symposium relies on the SM, such as the weak interactions that are critical to the r-process or Big Bang Nucleosynthesis. It is easy to forget, however, that the SM itself might not be what it is without the pioneering work of nuclear physicists. It has now been more than fifty years since the observation of parity violation in nuclear $\beta$-decay and over twenty-five years since parity-violating deep inelastic electron-deuteron scattering singled out the $\mathrm{SU}(2)_{L} \times \mathrm{U}(1)_{Y}$ prediction for the weak neutral current interaction from among other possibilities. Precise nuclear physics have provided other tests of the SM - such as SM predictions for the absence of second class currents - while providing some of the most precise inputs into the SM Lagrangian. For example, the extensive program of studies of superalllowed nuclear $\beta$-decay have yielded the most precise value of any element of the Cabibbo-KobayashiMaskawa matrix $\left(V_{\mathrm{ud}}\right)$, while searches for the permanent electric dipole moment (EDM) of the neutron have told us that the coefficient of the CP-violating " $\theta$ term" in the QCD Lagrangian is vanishingly small, pointing to the Peccei-Quinn symmetry and the possible existence of axions.

These successes notwithstanding, there exist important observations for which the SM provides no explanation. From my perspective, the most puzzling involve cosmology. Assuming the universe was matter-antimatter symmetric at the end of inflation, the SM interactions cannot account for the next excess of matter observed today. The SM has no candidate for the cold dark matter, and it provides no clue about the dark energy responsible for cosmic acceleration. The observation of neutrino oscillations also points to physics beyond the SM. During the coming decade, we hope to arrive at a clearer

CP1265, VIII Latin American Symposium on Nuclear Physics and Applications edited by R. Alcaron, H. F. Arellano, P. L. Cole, and A. J. Kreiner

(C) 2010 American Institute of Physics 978-0-7354-0814-2/10/\$30.00 
picture of the larger theory in which the SM is embedded - what I will call the "new Standard Model" (NSM). The quest to uncover the NSM requires experimental and theoretical studies at both the high energy frontier (i.e., the LHC) and the high precision frontier. In this talk I will concentrate on the latter and argue that nuclear physics has a vital role to play.

In so doing, I will concentrate on three types of studies: searches for the permanent electric dipole moments (EDMs) of various systems; searches for the neutrinoless double beta decay $(0 v \beta \beta)$ of atomic nuclei; and precision measurements of processes that are allowed in the SM. For a discussion of other topics, please refer to my recent reviews[1,2].

\section{ELECTRIC DIPOLE MOMENT SEARCHES}

The most sensitive test of CP symmetry in flavor conserving interactions is the search for a static EDM. In the SM, one expects a non-vanishing EDM due to the CP-violation (CPV) in the CKM matrix, but the expected magnitude lies well below the current and prospective sensitivity of EDM searches. The suppression of the SM CKM-based expectations occurs because this origin of EDMs requires two flavor changing vertices and is known to start off, for an elementary fermion, at three-loop order. On the other hand, the possible size of an EDM arising from another source could be as large as the present limits. In the case of CPV associated with the QCD $\theta$-term, the naive theoretical prejudice is that it should lead to an EDM of the neutron that is roughly $10^{10}$ times larger than the present limit. The stringent $d_{n}$ limit presents a puzzle as to why the corresponding parameter $\bar{\theta}$ is so tiny. One solution - spontaneously broken Peccei-Quinn symmetry - may help explain the dark matter mystery in the guise of the associated axion. If so, it may be that the observation of a neutron or mercury EDM associated with $\bar{\theta}$ is just around the corner.

On the other hand, if an observable EDM is associated with new physics rather than the massless gluons of QCD, then the effects of the new CPV will scale as $1 / M^{2} \times$ $\sin \phi_{\mathrm{CPV}}$, where $M$ is the mass scale associated with the new particles and $\phi_{\mathrm{CPV}}$ is a CPV phase. Assuming that the corresponding EDM arises at one-loop order, then using naive dimensional analysis we see that the present limits imply that these experiments are probing either very high mass scales or very small phases:

$$
\sin \phi_{\mathrm{CPV}} \sim 1 \rightarrow M>5000 \mathrm{GeV} \quad \text { or } \quad M<500 \mathrm{GeV} \rightarrow \sin \phi_{\mathrm{CPV}}<10^{-2} .
$$

Future experiments hope to improve the sensitivities by factors of as much as 100 over the next several years, implying probes of physics at the $50 \mathrm{TeV}$ scale or phases at the $10^{-4}$ level. Either way, these experiments will have a reach extending well beyond that of the LHC and will search for CPV effects that are too small to be pulled out of the hadronic collider environment.

EDM searches are also important for addressing one of the outstanding problems in cosmology: the origin of the visible matter of the universe. If the universe was matterantimatter symmetric at the end of inflation, the particle physics of the subsequently evolving cosmos would have to be responsible for generating the cosmic baryon asymmetry. Four decades ago, Sakharov identified the essential ingredients needed for this 
to occur[3]: violation of baryon number conservation; violation of both $\mathrm{C}$ and $\mathrm{CP}$ conservation; and a departure from equilibrium dynamics, assuming that $\mathrm{CPT}$ is conserved. Although we do not know when these criteria were satisfied in the early universe, one attractive possibility is that baryogenesis took place during the electroweak symmetry breaking era, about 10 picoseconds after the Big Bang. In "electroweak baryogenesis" (EWB), a first order phase transition occurs during which bubbles of broken electroweak symmetry nucleate in a symmetric background. CPV interactions at the bubble wall lead to a net density of left-handed fermions that diffuses ahead of the wall, where electroweak sphalerons convert it into baryon number. The expanding bubbles capture this baryon number by quenching the sphalerons. This process will generate the observed baryon-to-photon ratio provided that (1) sufficiently large CPV asymmetries are generated during the transition and (2) the transition is strongly first order in order to quench the sphalerons after baryon number is created.

EWB is not viable in the SM even though it contains, in principle, all the necessary ingredients. The effects of CKM CPV are too weak to generate the large left-handed particle density needed to drive the sphalerons. Moreover, it is now known that electroweak symmetry breaking in the SM proceeds via a cross-over transition rather than a first order or even second order transition because the LEP lower bound on the SM Higgs mass is too high to allow a phase transition to occur. Consequently, if EWB is responsible for the observed baryon asymmetry, new electroweak physics is needed. This physics could be accessible at both the LHC and in EDM searches, making it the most testable baryogenesis scenario.

In order to confront the results of EDM with the observed baryon asymmetry, one needs robust computations of both EDMs and the early universe CPV asymmetries generated by new interactions. Computing the latter is particularly challenging, requiring the use of tools in non-equilibrium, finite temperature field theory. During the past several years, our group has made substantial progress in carrying out these computations, and we believe we have developed a more robst theoretical framework for calculating the baryon asymmetry than one had previously (see, e.g., Ref. [4] and references therein).

To illustrate the complexity of the computations, consider one attractive scenario for the NSM: supersymmetry. In the Minimal Supersymmetric Standard Model (MSSM), loop effects associated with scalar superpartners of the right handed (RH) top quark can lead to a strong first order phase transition compatible with the LEP bounds if the RH stop mass is less than about $125 \mathrm{GeV}$ [5]. In addition, the MSSM contains a plethora of new CPV phases whose effects are not suppressed by light quark Yukawa couplings as in the SM. The most important CPV phase involves the interactions of the fermionic superpartners of the Higgs bosons ("Higgsinos") and gauge bosons ("gauginos"). During the electroweak phase transition, the corresponding interactions lead to a nonvanishing density of Higgsinos and Higgs bosons that diffuse in front of the expanding electroweak bubbles. Particle number changing reactions are responsible for transferring this density into a net density of left-handed quarks and leptons that cause the sphalerons to create baryons. The complicated chain of reactions that mediates this transfer involves a system of order thirty coupled quantum Boltzmann equations. We have derived and solved this system of equations, taking into account the MSSM parameter-dependence of all the particle number changing reaction rates that enter. The result is a small but significant "wake" of left-handed SM fermions that diffuses ahead of the bubble to drive 
the sphalerons into action. The precise magnitude of the wake depends on both the CPV "kick" that occurs at the bubble wall and on the interplay of all the particle number changing reactions.

EDM limits place severe constraints on the size of the CPV kick and, thus, on the viability of supersymmetric EWB. In a scenario where the one-loop contributions dominate, EWB in the MSSM is marginally viable, if at all. For superpartners with masses below a $\mathrm{TeV}$, the generic bounds on the phases of $\sim 10^{-2}$ preclude the production of large CPV asymmetries during an electroweak phase transition. On the other hand, taking the superpartner masses to be several $\mathrm{TeV}$ in order to allow for larger $\mathrm{CPV}$ phases implies that they would decouple from the plasma during the electroweak phase transition, thereby having no impact on the production of left-handed fermion number density. The way around this seeming conundrum is to consider a scenario in which the scalar superpartners of the first and second generation fermions are heavy while the gauginos and higgsinos remain light. In this case, one can suppress the one-loop EDMs and in principle allow for larger CPV phases. Moreover, the light gauginos and higgsinos remain active during the phase transition, and it is their CPV interactions that could ultimately drive the MSSM EWB. One still has to content with EDM limits, however, because the light gauginos and higgsinos lead to important two-loop EDM contributions through the well-known "Barr-Zee" diagrams.

Recently, we completed a computation of these two-loop graphs[6, 7] and found that the resulting EDM limits on the phases relevant to EWB can still be relatively severe. In particular, electron and neutron EDM limits imply that the phase $\phi_{2} \equiv \operatorname{Arg}\left(\mu M_{2} b^{*}\right)$ associated with the supersymmetric $\mu$ parameter, the soft wino mass parameter $M_{2}$, and the soft Higgs mass parameter $b$ is too small to lead to successful EWB. On the other hand, two-loop EDMs depend much less strongly (by about a factor of fifty) on the phase $\phi_{1} \equiv \operatorname{Arg}\left(\mu M_{1} b^{*}\right)$ involving the soft bino mass parameter. Consequently, the most viable path to MSSM EWB involves CPV bino-higgsino interactions during the phase transition, while those involving winos are unlikely to play a significant role. Moreover, EDM limits imply that the SUSY-breaking mechanism must be non-universal - in contrast to, say, mSUGRA - so that $\phi_{1}$ and $\phi_{2}$ are independent parameters.

It is interesting to note that successful supersymmetric EWB also appears to be viable only in a region of gaugino-Higgsino mass parameter space that lies outside the reach of LHC searches for these particles. This situation illustrates a central theme of this talk: studies at both the precision and energy frontier are needed to determine whether the NSM involves electroweak scale physics that can address open problems in cosmology. In the case of EDMs, the physics reach may even exceed that of the LHC, though ideally we will witness discovery at both of these frontiers in the coming decade - discoveries that may help us solve the origin of matter problem.

\section{NEUTRINOLESS DOUBLE BETA DECAY}

An attractive alternative to EWB - and one that typically involves new physics at much higher energy scales (or earlier times) - is leptogenesis. The standard leptogenesis scenario requires lepton number violation, associated with the existence of a Majorana mass term for the neutrinos. The theoretically appealing see-saw paradigm for explaining the 
tiny scale of $m_{v}$ is also implies a Majorana mass term, making leptogenesis a "natural" baryogenesis scenario.

A powerful way to search for lepton number violation is to search for $0 v \beta \beta$. Assuming that the decay proceeds by the exchange of a virtual light Majorana neutrino, then the rate is proportional to the square of the effective mass $m_{\beta \beta}$ that is not in general identical with the mass of the lightest neutrino, $m_{1}$. The relationship between the two involves the two possible Majorana phases and depends on the neutrino mass hierarchy. For the quasi-degenerate or normal hierarchies, $m_{\beta \beta} \sim m_{1}$, while for the inverted hierarchy, $m_{\beta \beta}$ can be about an order of magnitude larger than $m_{1}$. The present generation of $0 v \beta \beta$ experiments will be able to probe for a signal associated with $m_{\beta \beta}$ of tens of meV, corresponding to the inverted hierarchy range. The normal hierarchy implies a much smaller effective mass while in the quasi-degenerate hierarchy it is much larger. The next generation of $0 v \beta \beta$ searches will be sensitive to light Majorana neutrino-mediated decays if the neutrino spectrum is either quasi-degenerate or inverted. Although the observation of a signal in these searches would not prove that leptogenesis is the dominant baryogenesis scenario, it would add strong weight to this possibility.

It is also interesting to ask what the absence of an observation might mean. If combined with the results from other neutrino experiments, one might be able to conclude that neutrinos are Dirac particles. For example, the KATRIN experiment is measuring the $\beta$ spectrum of tritium $\beta$-decay, looking for behavior at the endpoint that might indicate a non-zero value of $m_{1}$. Given the sensitivity of this experiment, if an endpoint deviation is observed, it would tell us the absolute scale of neutrino mass (something oscillation experiments alone cannot do) and imply that the spectrum is quasi-degenerate. If neutrinos are Majorana particles, one would then expect a non-zero result in the present $0 v \beta \beta$ experiments. The absence of an observation could imply that neutrinos are Dirac. If KATRIN obtains a null result, but future long baseline experiments indicate an inverted hierarchy, one could again expect a signal in the $0 v \beta \beta$ searches and conclude from the absence of observation that we have light Dirac neutrinos.

There exists an important loophole in this scenario, however. It is possible that the mechanism responsible for $0 v \beta \beta$ is not the exchange of a light Majorana neutrino but rather the exchange of one or more heavy particles that entail lepton number violating (LNV) interactions (see, e.g., Ref. [8] and references therein) . In R parity-violating (RPV) SUSY, for example, the decay can proceed via the LNV $L Q \bar{D}$ operators and the exchange of the Majorana gluino. Similarly, left-right symmetric models with heavy right-handed Majorana neutrinos can lead to the same situation. If the mass scale of the heavy particles involved in the exchange is at the $\mathrm{TeV}$ scale, then the corresponding $0 v \beta \beta$ amplitude can be comparable to the expected amplitude associated with light Majorana neutrino exchange. Until we are certain as to the dominant mechanism, we cannot conclusively interpret either a signal or limit without making an additional assumption as to the mechanism. In effect, the nucleus that decays in $0 v \beta \beta$ is like a "black box" whose output can tell us that lepton number is violated but cannot easily indicate the way in which this violation occurs.

It may be possible to use information from other studies, such as those looking for charged lepton flavor violation - as well as the comparison of decays from a variety of isotopes - as diagnostic tools for determining the mechanism for $0 v \beta \beta$. My collaborators and I have carried out some exploratory investigations in the former possibility, but 
there clearly exists room for more theoretical work in this regard. From the standpoint of theory, perhaps a more familiar challenge is that of carrying out robust computations of the nuclear matrix elements for the decay. Since my graduate student days twenty years ago, nuclear theorists have been contending with this problem. There has been some progress. Recently, it has been shown that for QRPA computations, if one "calibrates" the parameter $g_{p p}$ to reproduce the observed two neutrino decay rates, the spread of QRPA predictions for the neutrinoless decay matrix elements is considerably reduced (see e.g., Ref. [9]). On the other hand, there remains the ongoing problem of determining whether QRPA or nuclear shell model computations come closer to giving the correct result for the matrix element. I hope that with the plans for construction of FRIB in the United States, there will be an infusion of energy into the nuclear structure community that will lead to a breakthrough on this problem. Theory challenges notwithstanding, however, the observation $0 v \beta \beta$ would imply without question that neutrinos are Majorana particles, as shown by Schecter and Valle[10]. Such a discovery would be in an of itself a landmark result in the quest for the NSM.

\section{PRECISION TESTS}

The searches for EDMs and $0 v \beta \beta$ involve processes that are highly suppressed or forbidden in the SM. Considerable experimental and theoretical efforts are also being devoted to nuclear physics studies of observables that are not forbidden, such ordinary nuclear $\beta$-decay, neutron decay, and pion decay. Any convincing deviation from a SM prediction for such an observable could point to new physics. More generally the pattern of deviations, or lack thereof, from a variety of these "precision tests" provide important guidance into the nature of the NSM. I will illustrate the power of these precision tests with parity-violating electron scattering (PVES), a process that has lead to a substantial experimental program at Jefferson Laboratory and is familiar to many people at this meeting.

The PVES frontier during the next decade involves scattering of longitudinally polarized electrons from fixed targets, such as protons or electrons in hydrogen. One measures the PV asymmetry for scattering involving positive and negative helicity electrons (for reviews, see Refs. $[11,12,13])$ :

$$
A_{\mathrm{PV}}=\frac{N_{+}-N_{-}}{N_{+}+N_{-}}=\frac{G_{F} Q^{2}}{4 \sqrt{2} \pi \alpha}\left[Q_{W}+F\left(Q^{2}\right)\right],
$$

where $N_{+}\left(N_{-}\right)$are the number of counts for positive (negative) helicity electrons. The quantity of interest for new physics is the weak charge of the target, $Q_{W}$, while the case of hadronic/nuclear targets, the form factor $F\left(Q^{2}\right)$ has been under intensive scrutiny for two decades as a way of probing the strange quark contributions to the nucleon's electromagnetic structure. The weak charge and $F\left(Q^{2}\right)$ can be experimentally separated by exploiting the $Q^{2}$-dependence of the latter.

The weak charges of the proton and electron are particularly interesting as a window on new physics, as both are proportional to $1-4 \sin ^{2} \theta_{W}$ (at tree level). Due to the near cancellation between the two terms in this expression, the SM predictions for $Q_{W}^{e, p}$ are suppressed, leading to a relatively enhanced sensitivity to new physics. For the same 
reason, a precise measurement of $Q_{W}^{e, p}$ can provide a precise determination of the weak mixing angle. This feature motivated the recently completed E158 PV Moller scattering experiment at SLAC and provides part of the rationale for the upcoming Q-Weak experiment and future PV Moller experiment at Jefferson Laboratory. A determination of $\sin ^{2} \theta_{W}$ in these experiments is interesting, even in light of the per mil accuracy of $\sin ^{2} \theta_{W}$ determinations at LEP and the SLC, because the SM predicts that the weak mixing angle runs with energy scale. Apart from looking for new physics, the low-energy PVES experiments provide a test of this predicted running, as discussed in detail in Refs. [14, 15].

To illustrate the new physics sensitivity of the future PVES experiments, consider again the MSSM. In this scenario, $Q_{W}^{e, p}$ can deviate from the SM expectations (taking the Z-pole weak mixing angle as input and using its SM running) due to two effects: loop corrections involving superpartners and tree-level contributions to the PV amplitudes arising from RPV interactions. My collaborators and I have computed these corrections $[16,2]$ and have found that a comparison of the weak charges can provide an interesting diagnostic tool for SUSY if it is discovered at the LHC. In particular, the signatures of MSSM loops and RPV interactions on these two weak charges are rather distinctive. In this context, the presence of RPV interactions would imply a Majorana mass for the light neutrinos. Moreover, it would preclude a neutralino dark matter candidate in the MSSM. The central value of the E158 results lies in the RPV-favored region, but the error bars are too large to be anything more than suggestive. On the other hand, the muon $g-2$ favors light superpartners - a region of MSSM parameter space that favors the larger MSSM loop corrections to the weak charges. It will be interesting to see if the PVES results favor one direction or the other - or an altogether different new physics scenario.

\section{CONCLUSIONS}

I hope I have illustrated how studies at the precision frontier in nuclear physics are an essential complement to the LHC in searching for the symmetries of the NSM. Searches for SM-forbidden or suppressed phenomena, such as the EDM or $0 v \beta \beta$, could reveal violations of fundamental symmetries that are needed to explain the origin of matter, while precision tests such as PVES and weak decays can probe detailed aspects of potential new forces. I am convinced that there is a rich potential for both discovery and insight about the NSM with nuclear physics, and look forward to what the combination precision studies and collider searches may teach us in the next decade about nature's fundamental interactions.

\section{ACKNOWLEDGMENTS}

I wish to thank the organizers of the Latin American Nuclear Physics Symposium for their hospitality. This work was supported in part by the U.S. Department of Energy contract DE-FG02-08ER41531 and the Wisconsin Alumni Research Foundation. 


\section{REFERENCES}

1. J. Erler and M. J. Ramsey-Musolf, Prog. Part. Nucl. Phys. 54, 351 (2005) [arXiv:hep-ph/0404291].

2. M. J. Ramsey-Musolf and S. Su, Phys. Rept. 456, 1 (2008) [arXiv:hep-ph/0612057].

3. A. D. Sakharov, Pisma Zh. Eksp. Teor. Fiz. 5, 32 (1967) [JETP Lett. 5, 24 (1967 SOPUA,34,392393.1991 UFNAA, 161,61-64.1991)].

4. D. J. H. Chung, B. Garbrecht, M. J. Ramsey-Musolf and S. Tulin, JHEP 0912, 067 (2009) [arXiv:0908.2187 [hep-ph]].

5. M. Carena, G. Nardini, M. Quiros and C. E. M. Wagner, Nucl. Phys. B 812, 243 (2009) [arXiv:0809.3760 [hep-ph]].

6. Y. Li, S. Profumo and M. Ramsey-Musolf, Phys. Rev. D 78, 075009 (2008) [arXiv:0806.2693 [hep$\mathrm{ph}]$.

7. Y. Li, S. Profumo and M. Ramsey-Musolf, Phys. Lett. B 673, 95 (2009) [arXiv:0811.1987 [hep-ph]].

8. V. Cirigliano, A. Kurylov, M. J. Ramsey-Musolf and P. Vogel, Phys. Rev. Lett. 93, 231802 (2004) [arXiv:hep-ph/0406199].

9. V. A. Rodin, A. Faessler, F. Simkovic and P. Vogel, Nucl. Phys. A 766, 107 (2006) [Erratum-ibid. A 793, 213 (2007)] [arXiv:0706.4304 [nucl-th]].

10. J. Schechter and J. W. F. Valle, Phys. Rev. D 25, 2951 (1982).

11. M. J. Musolf, T. W. Donnelly, J. Dubach, S. J. .. Pollock, S. Kowalski and E. J. Beise, Phys. Rept. 239, 1 (1994)

12. D. H. Beck and B. R. Holstein, Int. J. Mod. Phys. E 10, 1 (2001) [arXiv:hep-ph/0102053].

13. E. J. Beise, M. L. Pitt and D. T. Spayde, Prog. Part. Nucl. Phys. 54, 289 (2005) [arXiv:nuclex/0412054].

14. A. Czarnecki and W. J. Marciano, Int. J. Mod. Phys. A 15, 2365 (2000) [arXiv:hep-ph/0003049].

15. J. Erler and M. J. Ramsey-Musolf, Phys. Rev. D 72, 073003 (2005) [arXiv:hep-ph/0409169].

16. A. Kurylov, M. J. Ramsey-Musolf and S. Su, Phys. Rev. D 68, 035008 (2003) [arXiv:hep$\mathrm{ph} / 0303026]$. 